\title{
ROOTS OF IRREDUCIBLE POLYNOMIALS IN TAME HENSELIAN EXTENSION FIELDS
}

\author{
RON BROWN \\ Department of Mathematics \\ 2565 McCarthy Mall \\ University of Hawaii \\ Honolulu, HI 96822, USA \\ (email: ron@math.hawaii.edu)
}

\begin{abstract}
A class of irreducible polynomials $\mathcal{P}$ over a valued field $(F, v)$ is introduced, which is the set of all monic irreducible polynomials over $F$ when $(F, v)$ is maximally complete. A "best-possible" criterion is given for when the existence of an approximate root in a tamely ramified Henselian extension $K$ of $F$ to a polynomial $f$ in $\mathcal{P}$ guarantees the existence of an exact root of $f$ in $K$.
\end{abstract}

\section{$\S 1$. Introduction and the main theorem}

Throughout this paper $(F, v)$ will be a valued field with nontrivial value group $v F$, residue class field $\bar{F}$ and valuation $\operatorname{ring} \mathcal{O}$. For any $a \in \mathcal{O}$ and polynomial $h \in \mathcal{O}[x]$ we let $\bar{a}$ and $\bar{h}$ denote the canonical images of $a$ and $h$ in $\bar{F}$ and $\bar{F}[x]$, respectively.

We begin with an example. Suppose that $q$ is an odd prime. Then $f(x)=x^{2}-q$ is irreducible over the $q$-adic numbers $\mathbb{Q}_{q}$, and if $K$ is an algebraic extension of $\mathbb{Q}_{q}$ with an element $\alpha$ with $v(f(\alpha))>v(q)$ (where $v$ is the $q$-adic valuation), then $2 v\left(f^{\prime}(\alpha)\right)=v(q)$ and hence by Hensel's Lemma (Engler and Prestel, 2005, Theorem 1.3.1) a root of $f$ lies

2000 Mathematics Subject Classification. Primary: 12J20, 12 E05.

Key words and phrases. Henselian field, irreducible polynomial, tame ramification, root of a polynomial. 
in $K$. More generally suppose that $g \in F[x]$ is a generalized Schönemann polynomial over $F$, i.e., that: $g=p^{e}+t h$ where $e \geq 1 ; p \in \mathcal{O}[x]$ is monic with $\bar{p}$ irreducible; $h \in \mathcal{O}[x]$ has $\bar{p} \nmid \bar{h}$ and $\operatorname{deg} p^{e}>\operatorname{deg} h$; and $t \in \mathcal{O}$ has $v(t) \notin s v F$ for all divisors $s>1$ of $e$. Then $g$ is also irreducible over $F$, and if $(F, v)$ is Henselian and if a tamely ramified finite degree extension $(K, u)$ of $(F, v)$ has an element $\alpha$ with $u(g(\alpha))>v(t)$, then $K$ has a root of $g$ (Brown, 2007, Lemma 4 and Corollary 7).

In this paper we generalize the above result to a large class $\mathcal{P}$ of irreducible polynomials over $F$. If $v$ is discrete rank one, then $\mathcal{P}$ is the set of monic polynomials over $F$ which are irreducible over the completion of $(F, v)$, and if $(F, v)$ is maximally complete (Schilling, 1950, Definition 9, p. 36), then $\mathcal{P}$ is the set of all monic irreducible polynomials over $F$. (See Remark 6(C) below.) The role of " $v(t)$ " in this generalization will be played for each $h \in \mathcal{P}$ by an invariant $\gamma_{h}$ of $h$ lying in the divisible hull of $v F$ which will be constructed along with the set $\mathcal{P}$, but which can be characterized intrinsically in several ways. (See Remark 6(B).) The construction and analysis of $\mathcal{P}$ and the invariants $\gamma_{h}$ will be made in the next section. Anticipating that work, we close this introduction by stating our only theorem. It will be proved in Section 3.

Theorem 1. Suppose that $(F, v)$ is Henselian and $h \in \mathcal{P}$. Suppose that $\alpha$ is an element of a tamely ramified finite degree extension $(K, u)$ of $(F, v)$ with $u(h(\alpha))>\gamma_{h}$. Then there is a root of $h$ in $K$.

The analysis of $\gamma_{h}$ will show that for each $h \in \mathcal{P}$ it is best possible in the above theorem (see Remark 8 at the end of Section 3 ). The hypothesis that $(K, u)$ is a tamely ramified extension of $(F, v)$ says that the field extension $\bar{K} / \bar{F}$ is separable, the characteristic of $\bar{F}$ 
does not divide the ramification index $e_{u / v}=(u K: v F)$, and $[K: F]$ is the product of $e_{u / v}$ and the residual degree $f_{u / v}=[\bar{K}: \bar{F}]$.

There are examples in (Brown, 2007, Remark 2(B)) showing that Theorem 1 gives a stronger result in general than those obtained from some natural direct applications of Hensel's Lemma to the problem of the existence of roots of polynomials in $\mathcal{P}$.

\section{$\S 2$. The class $\mathcal{P}$ of polynomials}

We shall let $\mathcal{E}$ denote the set of all extensions of $v$ to a valuation on $F[x]$ mapping into $\mathbb{Q} v F \bigcup\{\infty\}$ where we let $\mathbb{Q} v F$ denote a (fixed) divisible hull of $v F$. For extensions $w$ of $v$ to a valuation on $F[x]$ we allow $w^{-1}(\infty)$ to be a nonzero ideal of $F[x]$. The extensions $w$ with $w^{-1}(\infty)$ trivial correspond precisely to extensions of $v$ to the rational function field $F(x)$, and the extensions with $w^{-1}(\infty)$ nontrivial (in which case $w^{-1}(\infty)$ is a maximal ideal) correspond precisely to the extensions of $v$ to the field $F[x] / w^{-1}(\infty)$. This paper is indeed about algebraic extensions of $F$; however, valuations on $F[x]$ give us a setting in which we can describe ways in which two extensions of $v$ to possibly different simple algebraic extensions of F can be closely related.

A notational convention will be useful. Whenever $w$ (respectively, $w_{i}$ ) is used to denote an extension of $v$ to a valuation on $F[x]$, we will denote the associated (surjective) place by $\tau: F[x] \longrightarrow k \bigcup\{\infty\}$ (respectively, by $\tau_{i}: F[x] \longrightarrow k_{i} \bigcup\{\infty\}$ ).

Definition 2. Suppose that $n \geq 0$. A strict system of polynomial extensions over $(F, v)$ of length $n+1$ is a finite sequence

$$
g=\left(\left(g_{0}, w_{0}, \gamma_{0}\right), \cdots,\left(g_{n+1}, w_{n+1}, \gamma_{n+1}\right)\right)
$$


of elements of $F[x] \times \mathcal{E} \times(\mathbb{Q} v F \bigcup\{-\infty\})$ such that for some $a \in F$,

(A) $g_{0}=x-a, \gamma_{0}=-\infty$, and $w_{0}(h)=v(h(a))$ for all $h \in F[x]$;

and when $0 \leq i \leq n$ :

(B) $\operatorname{deg} g_{i+1}>\operatorname{deg} g_{i}$;

(C) $\gamma_{i+1}=w_{i}\left(g_{i+1}\right)$;

(D) $w_{i+1}\left(g_{i+1}\right)=\infty$;

(E) $w_{i}\left(A_{r}\right) /\left(d_{i}-r\right) \geq w_{i}\left(A_{0}\right) / d_{i}>\gamma_{i}$ for all $r<d_{i}$ where

$$
g_{i+1}=g_{i}^{d_{i}}+\sum_{r<d_{i}} A_{r} g_{i}^{r}
$$

is the $g_{i}$-expansion of $g_{i+1}$ (so $\operatorname{deg} A_{r}<\operatorname{deg} g_{i}$ for all $r<d_{i}$ );

(F) if $e>0$ is least with $e w_{i}\left(A_{0}\right) \in d_{i} w_{i} F[x]$, then the polynomial

$$
Y^{d_{i} / e}+\sum_{r<d_{i} / e} \tau_{i}\left(s^{\left(d_{i} / e\right)-r} A_{e r}\right) Y^{r}
$$

is irreducible over $k_{i}$ for all $s \in F[x]$ with $d_{i} w_{i}(s)=-e w_{i}\left(A_{0}\right)$.

The symbol $-\infty$ above is used with the convention that $-\infty<a$ for all $a \in \mathbb{Q} v F \cup\{\infty\}$. One should observe that in (F) of the above definition we have $e \mid d_{i}$; indeed

$$
e=\left(w_{i} F[x]+\mathbb{Z} \frac{1}{d_{i}} w_{i}\left(A_{0}\right): w_{i} F[x]\right) .
$$

Also one can check that the irreducibility of the polynomial (1) above over $k_{i}$ is independent of the choice of $s$ and, using the inequalities of (E), that the coefficients of this polynomial are indeed finite.

We let $\mathcal{P}=\mathcal{P}(F)$ denote the set of all polynomials over $F$ which appear as the first coordinate of some term $(h, w, \gamma)$ of some strict system of polynomial extensions over $(F, v)$. 
That any such polynomial $h$ uniquely determines both the corresponding coordinates $w$ and $\gamma$ independently of any strict system of polynomial extensions in which $h$ appears will follow from Proposition 5 and Remark 6(B) below. Definition 2, while admittedly complicated, puts the notion of a sequence of $n+1$ extensions of polynomials from (Brown, 1972, Definition 5.8, p. 467, and $\S 7$ ) (with the degrees of the polynomials strictly increasing and the first polynomial linear) into a setting which allows an efficient inductive analysis in Proposition 5 of the polynomials in $\mathcal{P}$ and their associated valuations and invariants. While this paper uses ideas and some arguments from (Brown, 1972), we will not use the results of that paper except in Remark 6(C), which itself is not applied elsewhere in this paper.

Examples 3. Suppose that $g=p^{e}+t h \in F[x]$ is a generalized Schönemann polynomial as in Section 1.

(A) If $\operatorname{deg} p=1$ and $e>1$, then $\left(\left(p, u_{0},-\infty\right),\left(g, u_{1}, v(t)\right)\right)$ is a strict system of polynomial extensions of length one, where $u_{0}\left(\sum b_{i} p^{i}\right)=v\left(b_{0}\right)$ and $u_{1}\left(c g+\sum_{i<e} b_{i} p^{i}\right)=$ $\min _{i}\left(v\left(b_{i}\right)+\frac{i}{e} v(t)\right)$ for all $b_{i} \in F, c \in F[x]$. A concrete example over the field $\mathbb{Q}_{3}$ of 3-adic numbers of such a polynomial $g$ would be $(x-1)^{2}-3$.

(B) If $\operatorname{deg} p>1$, then $\left(\left(x, w_{0},-\infty\right),\left(p, w_{1}, 0\right)\right)$ is a strict system of polynomial extensions of length one, where $w_{0}(c)=v(c(0))$ and $w_{1}\left(p c+\sum_{i<\operatorname{deg} p} b_{i} x^{i}\right)=\min _{i<\operatorname{deg} p} v\left(b_{i}\right)$ for all $c \in F[x], b_{i} \in F$.

(C) If $\operatorname{deg} p>1$ and $e>1$, then $\left(\left(x, w_{0},-\infty\right),\left(p, w_{1}, 0\right),\left(g, w_{2}, v(t)\right)\right)$ is a strict system of polynomial extensions of length 2 , where $w_{2}\left(c g+\sum_{i<e} b_{i} p^{i}\right)=\min _{i}\left(w_{1}\left(b_{i}\right)+\frac{i}{e} v(t)\right)$ for all $c \in F[x]$ and $b_{i} \in F[x]$ with $\operatorname{deg} b_{i}<\operatorname{deg} p$. An example of such a polynomial $g$ over $\mathbb{Q}_{3}$ 
would be the classical Schönemann polynomial $\left(x^{2}-2\right)^{2}-3$.

The above examples generalize routinely to a class of polynomials considered by Khanduja and Saha (1997, Theorem 1.1) even without the separability requirement of their theorem.

Let $N \geq 0$. Our last two examples will be strict systems of polynomial extensions of length $N+1$. Both will have the form

$$
g=\left(\left(g_{0}, w_{0},-\infty\right),\left(g_{1}, w_{1}, \gamma_{1}\right), \cdots,\left(g_{N+1}, w_{N+1}, \gamma_{N+1}\right)\right)
$$

where for each $n$ we denote by $w_{n}$ an extension of $v$ to a valuation on $F[x]$ with $w_{n}\left(g_{n}\right)=\infty$.

(D) Let $p$ be a rational prime and $v$ be the $p$-adic valuation on $\mathbb{Q}$ with $v(p)=1$. Let $g_{0}=x, g_{1}=x^{2}-p$, and for each $n \geq 1$ let $g_{n+1}=g_{n}^{2}-p^{2^{n}} g_{n-1}$ and let $\gamma_{n}=\frac{4^{n}-1}{3} \frac{1}{2^{n-1}}$. Then (i) the sequence $g$ above is a strict system of polynomial extensions over $(\mathbb{Q}, v)$ of length $N+1$, and (ii) $w_{N+1}\left(g_{N}\right)=\gamma_{N+1} / 2$. The $N=0$ case of the assertion (i) is included in Example (A) above, and the general case of both assertions is easily proven by induction on $N$ using Proposition 5 below. (To prove (i) apply the Proposition to $w_{N}$, and having proved (i), then prove (ii) by applying the Proposition to $w_{N+1}$. The key observation is that the induction hypothesis implies that $w_{N}\left(g_{N+1}-g_{N}^{2}\right)=\gamma_{N+1}$. $)$ The Proposition implies that the $w_{n}$ are uniquely determined.

(E) Let $v$ be the $t$-adic valuation on the rational function field $\mathbb{Q}(t)$ with $v(t)=1$. Let $g_{0}=x, g_{1}=x^{2}-2$, and for all $n \geq 1$ let $g_{n+1}=g_{n}^{2}-t^{2^{n+1}-2^{n-1}-1} g_{n-1}$ and let $\gamma_{n}=2^{n}-2$. Then (i) $g$ is a strict system of polynomial extensions over $(\mathbb{Q}(t), v)$ of length $N+1$, and (ii) $g_{N} / t^{2^{N}-1}$ is a unit with respect to the valuation $w_{n+1}$ and its residue class is a $2^{N+1}$ st root of 2. The $N=0$ case of this assertion is a special case of Example (B) above, and 
the general case follows by induction on $N$ using exactly the outline given parenthetically in the previous example (D).

For the remainder of this section we assume that $g$ is a strict system of polynomial extensions over $(F, v)$ and use the notation for $g$ in the above Definition 2. In the next Proposition we will also use the convention introduced just before Definition 2 as well as the following

Notation 4. (A) Write $J_{0}=\{0\}$ and $g^{0}=1$. When $0<i \leq n+1$ let $J_{i}=\prod_{j<i}\left\{0,1, \ldots, d_{j}-\right.$ 1 \}. For any $\sigma \in \mathbb{Z}^{i}$ we write $0 \leq \sigma$ if all the coordinates of $\sigma$ are nonnegative, in which case we also write $\sigma=(\sigma(0), \cdots, \sigma(i-1))$ and $g^{\sigma}=\prod_{j<i} g_{j}^{\sigma(j)}$. In all cases, $\left\{g^{\sigma}: \sigma \in J_{i}\right\}$ is a basis for the $F$-space $F[x]_{\operatorname{deg} g_{i}}$, where for any $m \geq 1$ we write $F[x]_{m}=\{h \in F[x]: \operatorname{deg} h<m\}$. We will say that $\left\{g^{\sigma}: \sigma \in J_{i}\right\}$ is a valuation basis for $F[x]_{\operatorname{deg} g_{i}}$ with respect to an extension of $v$ to a valuation $w$ on $F[x]$ if for all choices of $a_{\sigma} \in F$ we have

$$
w\left(\sum_{\sigma \in J_{i}} a_{\sigma} g^{\sigma}\right)=\min _{\sigma \in J_{i}} w\left(a_{\sigma} g^{\sigma}\right) .
$$

(B) Let $E$ denote either $F[x]$ or a field extension of $F$. For any extension $u$ of $v$ to a valuation on $E$ let $e_{u / v}=(u E: v F)$ denote the ramification index and $f_{u / v}=[\bar{E}: \bar{F}]$ denote the residual degree of the extension.

Proposition 5. Suppose that $w$ is an extension of $v$ to a valuation on $F[x]$ with $w\left(g_{n+1}\right)>$ $\gamma_{n+1}$. Then for all integers $i$ with $0 \leq i \leq n$ we have:

(A) $w\left(g_{i}\right)=w_{i+1}\left(g_{i}\right)=\gamma_{i+1} / d_{i}$;

(B) $\left\{g^{\sigma}: \sigma \in J_{i+1}\right\}$ is a valuation basis for $w$ on $F[x]_{\operatorname{deg} g_{i+1}}$; 
(C) $g_{i+1}$ is irreducible over $F$; $w_{i+1}$ is the unique extension of $v$ to $F[x]$ mapping $g_{i+1}$ to $\infty ;$ and $\operatorname{deg} g_{i+1}=e_{w_{i+1} / v} f_{w_{i+1} / v} ;$

(D) there is an $\bar{F}$-homomorphism $\Phi_{i+1}: k_{i+1} \longrightarrow k$ with $\Phi_{i+1} \tau_{i+1}\left(c g^{\sigma}\right)=\tau\left(c g^{\sigma}\right)$ whenever $c \in F, 0 \leq \sigma \in \mathbb{Z}^{i+1}$, and $w\left(c g^{\sigma}\right) \geq 0$.

In $(\mathrm{A})$ of the above Proposition we have identified (as one can do, uniquely) the subgroup of elements of $w F[x]$ which have a nonzero multiple in $v F$ with a subgroup of our fixed divisible hull $\mathbb{Q} v F$ of $v F$. Part (C) above is equivalent to the assertion that if $u$ is an extension of $v$ to $F[\xi]$ where $\xi$ is a root of $g_{i+1}$, then $\operatorname{deg} g_{i+1}=[F[\xi]: F]=e_{u / v} f_{u / v}$.

Remarks 6. We note some consequences of the Proposition above.

(A) Suppose that $0 \leq i \leq n$. Since $w_{i+1}\left(g_{i+1}\right)=\infty$, the place $\tau_{i+1}$ maps $F[x]_{\operatorname{deg} g_{i+1}}$ onto $k_{i+1} \bigcup\{\infty\}$ and $w_{i+1}$ maps $F[x]_{\operatorname{deg} g_{i+1}}$ onto $w_{i+1} F[x] \bigcup\{\infty\}$. Combining (A), (B) and (D) of the Proposition shows that $w$ and $w_{i+1}$ agree on $F[x]_{\operatorname{deg} g_{i+1}}$ and that $\Phi_{i+1} \tau_{i+1}$ and $\tau$ agree on $F[x]_{\operatorname{deg} g_{i+1}}$.

(B) Part (C) of the above Proposition says that $g_{i+1}$ uniquely determines $w_{i+1}$ for all $i \leq n$. We now argue that $g_{i+1}$ also uniquely determines $\gamma_{i+1}$. Specifically, $\gamma_{i+1}$ is the minimal element of $\mathbb{Q} v F$ with the property that if $u$ and $u^{*}$ are extensions of $v$ to $F[x]$ with $u\left(g_{i+1}\right)>\gamma_{i+1}<u^{*}\left(g_{i+1}\right)$, then they agree on $F[x]_{\operatorname{deg} g_{i+1}}$. That $\gamma_{i+1}$ has this property follows from the remarks in the previous paragraph; $\gamma_{i+1}$ is the minimal element of $\mathbb{Q} v F$ with this property, since $w_{i}\left(g_{i+1}\right)=\gamma_{i+1}<w_{i+1}\left(g_{i+1}\right)$, but $w_{i}$ and $w_{i+1}$ do not agree on $g_{i} \in F[x]_{\operatorname{deg} g_{i+1}}$. Since $g_{i+1}$ uniquely determines $\gamma_{i+1}$, we can unambiguously denote $\gamma_{i+1}$ by $\gamma_{g_{i+1}}$ (independently of any strict system of polynomial extensions in which $g_{i+1}$ appears but of course depending on the choice of $\left.(F, v)\right)$. This is the notation used 
in the statement of Theorem 1. Another characterization of $\gamma_{i+1}$ can be adapted from (Brown, 1972, Proposition 5.6, p. 467): $\gamma_{i+1}$ is the minimal element of $\mathbb{Q} v F$ such that if $h \in F[x]_{\operatorname{deg} g_{i+1}}$ and $w_{i+1}(h)>\gamma_{i+1}$, then $g_{i+1}+h$ is irreducible. A third intrinsic characterization of $\gamma_{i+1}$ follows from the fact that it is best possible in Theorem 1 (see Remark 7 below).

(C) First suppose that $(F, v)$ is a maximally complete field (Schilling, 1950, p. 36). By the Proposition all the elements of $\mathcal{P}$ are monic irreducible over $F$. Conversely any monic irreducible polynomial $h$ over $F$ can be shown by the methods of (Brown, 1972) to be in $\mathcal{P}$. Basically one takes the (possibly transfinite) generating sequence of the augmented signature (Brown, 1972, Definition 7.5, p. 477) of the unique extension $w$ of $v$ to $F[x]$ with $w(h)=\infty$, and then deletes all terms whose degree is the same as that of a later term.

Next suppose that $(F, v)$ is discrete rank one, say with completion $(\tilde{F}, \tilde{v})$. Any strict system of polynomial extensions over $(F, v)$ lifts to one over $(\tilde{F}, \tilde{v})$ by just extending the valuations on $F[x]$ to extensions of $\tilde{v}$ to $\tilde{F}[x]$. Thus each element of $\mathcal{P}$ is irreducible over $\tilde{F}$. In fact, in this case $\mathcal{P}$ is exactly the set of monic polynomials over $F$ which are irreducible over $\tilde{F}$. The key fact here is that for any monic polynomial $h$ in $F[x]$ which is irreducible over $\tilde{F}$ there is a unique extension $w$ of $\tilde{v}$ to $\tilde{F}[x]$ with $w(h)=\infty$, and if we take the systems of representatives $A$ and $B$ of (Brown, 1972, §4) to be in $F$, then the generating sequence of the augmented signature of $w$ will be a sequence of polynomials in $F[x]$ with last term $h$.

The remainder of this section is devoted to a proof of the Proposition. The proof of part (A) especially borrows heavily from the proof of the Fundamental Lemma in Section 
8 of (Brown, 1972). We included these arguments here since it seemed unreasonable to ask the reader to extract them from (Brown, 1972), where the exposition involves complicated machinery, some of which assumes that $(F, v)$ is maximally complete. The proof will show that when $0 \leq i \leq n$, then the value group of $w_{i+1}$ is $w_{i} F[x]+\mathbb{Z}\left(\gamma_{i+1} / d_{i}\right)$ and the residue class field of $w_{i+1}$ is isomorphic to an extension of the residue class field of $w_{i}$ by a root of the polynomial (1) of Definition 2.

Given our strict system of polynomial extensions $g$, we set for each $i \leq n$ :

$$
q_{i}=\gamma_{i+1} / d_{i}, \quad e_{i}=\left(w_{i} F[x]+\mathbb{Z} q_{i}: w_{i} F[x]\right), \quad \text { and } \quad f_{i}=d_{i} / e_{i} .
$$

Note that $q_{0}>\gamma_{0}=-\infty$ (by Definition 2 applied with $i=0$ ) and that if $0 \leq i \leq n$, then with $A_{0}$ as in Definition 2 we have

$$
d_{i} q_{i}=\gamma_{i+1}=w_{i}\left(g_{i+1}\right)=w_{i}\left(A_{0}\right)>d_{i} \gamma_{i}
$$

(using the definition of $q_{i}$ and parts (C), (D) and (E) of Definition 2, respectively). Hence, if $0 \leq i \leq n$, then

$$
q_{i}>\gamma_{i} \text { and if } i<n \text {, then } q_{i+1}>d_{i} q_{i} .
$$

Finally, by Equation (2) for any $i \leq n$ the value of $e$ in Definition 2(F) is exactly the $e_{i}$ above.

By induction we may assume that the Proposition is true for all strict systems of polynomial extensions over $(F, v)$ of length less than $n+1$.

Our first task is to prove that $w\left(g_{i}\right)=q_{i}$ whenever $0 \leq i \leq n$. Just suppose that this is not true. Then there exists a smallest $t$ with $0 \leq t \leq n$ and $w\left(g_{t}\right) \neq q_{t}$. Hence 
$w\left(g_{i}\right)=q_{i}$ for all $i<t$, and by our induction hypothesis on $n$ and the inequality (5) we have $w\left(g_{i}\right) \leq \gamma_{i}<q_{i}$ whenever $t<i \leq n$.

Claim 1. If $w\left(g_{t}\right)<q_{t}$, then $w\left(g_{i+1}\right)=d_{i} w\left(g_{i}\right)$ whenever $t \leq i \leq n$.

Proof of Claim 1. Suppose that $t \leq i \leq n$. We may suppose by induction on $i$ that $w\left(g_{m+1}\right)=d_{m} w\left(g_{m}\right)$ whenever $t \leq m<i$. We use the notation of Definition 2(E). For each $r<d_{i}$ we can write

$$
A_{r}=\sum_{\sigma \in J_{i}} c_{r \sigma} g^{\sigma}
$$

for some $c_{r \sigma} \in F$. If $i>0$ we can apply our induction hypothesis on $n$ to the valuation $w_{i}$ to show that for each $r<d_{i}$

$$
w_{i}\left(A_{r}\right)=\min _{\sigma \in J_{i}}\left(v\left(c_{r \sigma}\right)+\sum_{m<i} \sigma(m) q_{m}\right) .
$$

Equation (6) is trivially true if $i=0$. Thus by Equation (4) above and Definition 2(E) for all $r$ and $\sigma$ we have

$$
\begin{aligned}
w\left(c_{r \sigma} g^{\sigma}\right) & \geq w_{i}\left(A_{r}\right)+\sum_{m<i} \sigma(m)\left(w\left(g_{m}\right)-q_{m}\right) \\
& \geq\left(d_{i}-r\right) q_{i}+\sum_{m<i} \sigma(m)\left(w\left(g_{m}\right)-q_{m}\right) .
\end{aligned}
$$

Now $w\left(g_{m}\right)=q_{m}$ for all $m<t$, and $w\left(g_{m}\right)<q_{m}$ whenever $t \leq m \leq i$. Hence for all $r<d_{i}$ 
and $\sigma \in J_{i}$ setting $\sigma(i)=r$ yields

$$
\begin{aligned}
& w\left(c_{r \sigma} g^{\sigma} g_{i}^{r}\right)=w\left(c_{r \sigma} g^{\sigma}\right)+r w\left(g_{i}\right) \\
& \geq d_{i} q_{i}+\sum_{m \leq i} \sigma(m)\left(w\left(g_{m}\right)-q_{m}\right) \\
& \geq d_{i} q_{i}+\sum_{t \leq m \leq i}\left(d_{m}-1\right)\left(w\left(g_{m}\right)-q_{m}\right) \\
& \quad=d_{i} w\left(g_{i}\right)+\left(q_{t}-w\left(g_{t}\right)\right)+\sum_{t \leq m<i}\left(q_{m+1}-d_{m} q_{m}\right)+\sum_{t \leq m<i}\left(d_{m} w\left(g_{m}\right)-w\left(g_{m+1}\right)\right) \\
& >d_{i} w\left(g_{i}\right)
\end{aligned}
$$

(since $q_{t}>w\left(g_{t}\right)$, and since by our induction hypothesis on $i$ and formula (5), the last summation above is zero and the penultimate one is nonnegative). Therefore

$$
w\left(g_{i+1}\right)=w\left(g_{i}^{d_{i}}+\sum_{r<d_{i}} \sum_{\sigma \in J_{i}} c_{r \sigma} g^{\sigma} g_{i}^{r}\right)=w\left(g_{i}^{d_{i}}\right)=d_{i} w\left(g_{i}\right),
$$

proving the claim.

If $w\left(g_{t}\right)<q_{t}$, then the above claim tells us that $w\left(g_{n+1}\right)=d_{n} w\left(g_{n}\right)<d_{n} q_{n}=\gamma_{n+1}$, a contradiction. Hence we may conclude that if $w\left(g_{t}\right) \neq q_{t}$, then $w\left(g_{t}\right)>q_{t}$. Thus by our induction on $n,\left\{g^{\sigma}: \sigma \in J_{t}\right\}$ is a valuation basis for $w$ on $F[x]_{\operatorname{deg} g_{t}}$.

Claim 2. $w\left(g_{t+1}\right)=d_{t} q_{t}$ and $t<n$.

Proof of Claim 2. Note that $w_{t}$ and $w$ agree on $F[x]_{\operatorname{deg} g_{t}}$ by induction on $n$. We can write $g_{t+1}=g_{t}^{d_{t}}+\sum_{r<d_{t}} A_{r} g_{t}^{r}$ where $A_{r} \in F[x]_{\operatorname{deg} g_{t}}$ for each $r$. If $r \neq 0$, then

$$
w\left(A_{r} g_{t}^{r}\right)=w_{t}\left(A_{r}\right)+r w\left(g_{t}\right)>\frac{d_{t}-r}{d_{t}} w_{t}\left(A_{0}\right)+r q_{t}=d_{t} q_{t}
$$

(cf. display (4)). But $w\left(g_{t}^{d_{t}}\right)=d_{t} w\left(g_{t}\right)>d_{t} q_{t}$ and $w\left(A_{0}\right)=w_{t}\left(A_{0}\right)=d_{t} q_{t}$, so $w\left(g_{t+1}\right)=$ $d_{t} q_{t}$, as claimed. Thus $t \neq n$ since otherwise $w\left(g_{n+1}\right)=d_{n} q_{n}=\gamma_{n+1}<w\left(g_{n+1}\right)$. This completes the proof of Claim 2 . 
Claim 3. If $t<i \leq n$, then $w\left(g_{i+1}\right)=d_{i} w\left(g_{i}\right)$.

Proof of Claim 3. We are of course assuming that $w\left(g_{t}\right) \neq g_{t}$ and hence that $n>t$ and $w\left(g_{t}\right)>q_{t}$. We my assume inductively that $w\left(g_{m+1}\right)=d_{m} w\left(g_{m}\right)$ whenever $t<m<i$. As in the proof of Claim 1 we write

$$
g_{i+1}=g_{i}^{d_{i}}+\sum_{r<d_{i}} \sum_{\sigma \in J_{i}} c_{r \sigma} g^{\sigma} g_{i}^{r} .
$$

Then for all $0 \leq r<d_{i}$ and $\sigma \in J_{i}$ we have (again setting $\sigma(i)=r$ )

$$
\begin{aligned}
w\left(c_{r \sigma} g^{\sigma} g_{i}^{r}\right) & \geq d_{i} q_{i}+\sum_{m \leq i} \sigma(m)\left(w\left(g_{m}\right)-q_{m}\right) \\
& \geq d_{i} q_{i}+\sum_{t<m \leq i}\left(d_{m}-1\right)\left(w\left(g_{m}\right)-q_{m}\right) \\
& =\sum_{t<m<i}\left(q_{m+1}-d_{m} q_{m}+d_{m} w\left(g_{m}\right)-w\left(g_{m+1}\right)\right) \\
& +q_{t+1}-w\left(g_{t+1}\right)+d_{i} w\left(g_{i}\right) \\
& >d_{i} w\left(g_{i}\right)=w\left(g_{i}^{d_{i}}\right)
\end{aligned}
$$

(the last inequality uses formula (5) and our induction hypotheses on $i$ and $n$ ). The claim follows immediately.

Since $n>t$, we can take $i=n$ in Claim 3 to obtain

$$
w\left(g_{n+1}\right)=d_{n} w\left(g_{n}\right) \leq d_{n} q_{n}=\gamma_{n+1}
$$

contradicting the hypothesis on $w$. Hence for all $i \leq n$ we have $w\left(g_{i}\right)=q_{i}$. Applying this result with $w_{i+1}$ in place of $w$ shows that $w_{i+1}\left(g_{i}\right)=q_{i}=\gamma_{i+1} / d_{i}$. Thus our induction hypothesis on $n$ implies (A) of the Proposition. In particular, $w\left(g_{n}\right)=q_{n}>\gamma_{n}$, so that 13 
parts (B), (C) and (D) of the Proposition are valid for all $i<n$ by our induction hypothesis on $n$. We now prove them for $i=n$.

Because $w_{n+1}\left(g_{n}\right)=w\left(g_{n}\right)=q_{n}>\gamma_{n}$, we may assume by our induction on $n$ that we have $\bar{F}$-homomorphisms $\Phi_{n}: k_{n} \longrightarrow k$ and $\Phi_{n}^{\prime}: k_{n} \longrightarrow k_{n+1}$ with $\Phi_{n} \tau_{n}\left(c g^{\sigma}\right)=\tau\left(c g^{\sigma}\right)$ and $\Phi_{n}^{\prime} \tau_{n}\left(c g^{\sigma}\right)=\tau_{n+1}\left(c g^{\sigma}\right)$ whenever $c \in F, 0 \leq \sigma \in \mathbb{Z}^{n}$, and $w\left(c g^{\sigma}\right) \geq 0$. As usual we write

$$
g_{n+1}=g_{n}^{d_{n}}+\sum_{r<d_{n}} A_{r} g_{n}^{r} .
$$

There exist $b \in F$ and $\mu \in J_{n}$ such that $w\left(s^{f_{n}} A_{0}\right)=0$ where $s=b g^{\mu}$ (if $n>0$ then this follows from (B) with $i=n-1)$. By Definition 2(F) the polynomial

$$
G=G(Y)=Y^{f_{n}}+\sum_{r<f_{n}} \tau_{n}\left(s^{f_{n}-r} A_{r e_{n}}\right) Y^{r}
$$

is irreducible over $k_{n}$, and hence $\Phi_{n}(G)$ is irreducible over $\Phi_{n}\left(k_{n}\right)$.

We now show that $\tau\left(s g_{n}^{e_{n}}\right)$ is a root of $\Phi_{n}(G)$. By hypothesis and formula (4)

$$
w\left(g_{n+1}\right)>\gamma_{n+1}=w\left(A_{0}\right)=-w\left(s^{f_{n}}\right),
$$

and if $0 \leq r<d_{n}$ and $e_{n} \nmid r$, then

$$
w\left(A_{r} g_{n}^{r}\right)>\frac{d_{n}-r}{d_{n}} w\left(A_{0}\right)+r q_{n}=d_{n} q_{n}=-w\left(s^{f_{n}}\right) .
$$

Hence

$$
w\left(\left(s g_{n}^{e_{n}}\right)^{f_{n}}+\sum_{r<f_{n}}\left(A_{r e_{n}} s^{f_{n}-r}\right)\left(s g_{n}^{e_{n}}\right)^{r}\right)=w\left(s^{f_{n}}\left(g_{n+1}-\sum^{\prime} A_{r} g_{n}^{r}\right)\right)>0
$$

where $\sum^{\prime}$ is the sum over all $r<d_{n}$ with $e_{n} \nmid r$. Thus $\tau\left(s g_{n}^{e_{n}}\right)$ is indeed a root of

$$
\tau\left(Y^{f_{n}}+\sum_{r<f_{n}} A_{r e_{n}} s^{f_{n}-r} Y^{r}\right)=\Phi_{n}(G)
$$


(The equality above holds by the choice of $\Phi_{n}$, since $\left\{g^{\sigma}: \sigma \in J_{n}\right\}$ is a valuation basis for $w$ on $F[x]_{\operatorname{deg} g_{n}}$ and hence each $A_{r e_{n}} s^{f_{n}-r}$ is a sum of integral elements of the form $c g^{\sigma}$ where $c \in F$ and $0 \leq \sigma \in \mathbb{Z}^{n}$.)

Replacing $w$ by $w_{n+1}$ and $\tau$ by $\tau_{n+1}$ in the above argument shows that the irreducible polynomial $\Phi_{n}^{\prime} G$ over $\Phi_{n}^{\prime} k_{n}$ has root $\tau_{n+1}\left(s g_{n}^{e_{n}}\right)$ in $k_{n+1}$. This shows that $f_{w_{n+1} / v} \geq$ $f_{w_{n} / v} f_{n}$. Moreover, it also shows that the unique $\bar{F}$-isomorphism $\psi_{0}: \Phi_{n}^{\prime}\left(k_{n}\right) \longrightarrow \Phi_{n}\left(k_{n}\right)$ with $\psi_{0} \Phi_{n}^{\prime}=\Phi_{n}$ lifts to an isomorphism $\psi: \Phi_{n}^{\prime}\left(k_{n}\right)\left[\tau_{n+1}\left(s g_{n}^{e_{n}}\right)\right] \longrightarrow \Phi_{n}\left(k_{n}\right)\left[\tau\left(s g_{n}^{e_{n}}\right)\right]$ taking $\tau_{n+1}\left(s g_{n}^{e_{n}}\right)$ to $\tau\left(s g_{n}^{e_{n}}\right)$ (after all, $\left.\psi_{0}\left(\Phi_{n}^{\prime}(G)\right)=\Phi_{n}(G)\right)$.

Since $w_{n}\left(g_{n}\right)=\infty$,

$$
w_{n} F[x]=w_{n}\left(F[x]_{\operatorname{deg} g_{n}}\right) \backslash\{\infty\}=v F+\sum_{i<n} \mathbb{Z} q_{i}
$$

(by induction on $n$, using (A) and (B) of the statement of the Proposition). By our proof of part (A) we have $w_{n+1} F[x] \supseteq w_{n} F[x]$. Hence $e_{w_{n+1} / v} \geq e_{n} e_{w_{n} / v}$ by the definition of $e_{n}$ in the display $(3)$.

Because $w_{n+1}\left(g_{n+1}\right)=\infty$, there is a monic irreducible factor $h$ of $g_{n+1}$ with $w_{n+1}(h)=$ $\infty$. Then $w_{n+1}$ induces an extension $u$ of $v$ to the quotient field $F[x] /(h)$ and we have

$$
\begin{aligned}
\operatorname{deg} g_{n+1} & \geq \operatorname{deg} h=[F[x] /(h): F] \\
& \geq e_{u / v} f_{u / v}=e_{w_{n+1} / v} f_{w_{n+1} / v} \\
& \geq e_{n} f_{n} e_{w_{n} / v} f_{w_{n} / v} \\
& =e_{n} f_{n} \operatorname{deg} g_{n}=\operatorname{deg} g_{n+1}
\end{aligned}
$$

(using our induction hypothesis on $n$ ). This shows that $g_{n+1}=h$ is irreducible and that

$$
\left[F[x] /\left(g_{n+1}\right): F\right]=\operatorname{deg} g_{n+1}=e_{w_{n+1} / v} f_{w_{n+1} / v}=e_{u / v} f_{u / v} .
$$


Thus $u$ is the unique extension of $v$ to $F[x] /\left(g_{n+1}\right)$, cf. (Engler and Prestel, 2005, Theorem 3.3.4). It follows that $w_{n+1}$ is the unique extension of $v$ to $F[x]$ with $w_{n+1}\left(g_{n+1}\right)=\infty$. This completes the proof of $(\mathrm{C})$ when $i=n$; it also shows that $k_{n+1}=\Phi_{n}^{\prime}\left(k_{n}\right)\left[\tau_{n+1}\left(s g_{n}^{e_{n}}\right)\right]$ and hence that our homomorphism $\psi$ has domain $k_{n+1}$. Thus in order to prove (D) when $i=n$ it suffices to show that $\psi \tau_{n+1}\left(c g^{\sigma}\right)=\tau\left(c g^{\sigma}\right)$ whenever $c \in F, 0 \leq \sigma \in \mathbb{Z}^{n+1}$, and $w\left(c g^{\sigma}\right)=v(c)+\sum_{j \leq n} \sigma(j) q_{j} \geq 0$. This is obvious if $w\left(c g^{\sigma}\right)>0$, so we suppose that $w\left(c g^{\sigma}\right)=0$. This condition implies that we can write $c g^{\sigma}=c g^{\rho} g_{n}^{e_{n} j}$ where $0 \leq \rho \in \mathbb{Z}^{n}$ and $0 \leq j \in \mathbb{Z}$. We can also write $w\left(s^{j}\right)=-w\left(d g^{\delta}\right)$ for some $d \in F$ and $\delta \in J_{n}$. Then

$$
\begin{aligned}
& \psi\left(\tau_{n+1}\left(s^{j} d g^{\delta}\right)\right) \psi\left(\tau_{n+1}\left(c g^{\sigma}\right)\right) \\
& =\psi \tau_{n+1}\left(c d g^{\delta+\rho}\right) \psi \tau_{n+1}\left(\left(s g_{n}^{e_{n}}\right)^{j}\right) \\
& =\psi_{0} \Phi_{n}^{\prime} \tau_{n}\left(c d g^{\delta+\rho}\right) \tau\left(\left(s g_{n}^{e_{n}}\right)^{j}\right) \\
& =\Phi_{n} \tau_{n}\left(c d g^{\delta+\rho}\right) \tau\left(\left(s g_{n}^{e_{n}}\right)^{j}\right) \\
& =\tau\left(c d g^{\delta+\rho}\left(s g_{n}^{e_{n}}\right)^{j}\right) \\
& =\psi\left(\tau_{n+1}\left(s^{j} d g^{\delta}\right)\right) \tau\left(c g^{\sigma}\right) \neq 0,
\end{aligned}
$$

so indeed $\psi \tau_{n+1}\left(c g^{\sigma}\right)=\tau\left(c g^{\sigma}\right)$, proving (D) for $i=n$.

It remains to prove (B) when $i=n$. Consider any nonzero polynomial

$$
B=\sum_{j<d_{n}} B_{j} g_{n}^{j} \in F[x]_{\operatorname{deg} g_{n+1}}
$$

where each $B_{j} \in F[x]_{\operatorname{deg} g_{n}}$. Using induction on $n$, it suffices to prove that

$$
w(B)=\min _{j<d_{n}} w\left(B_{j} g_{n}^{j}\right)
$$


We may without loss of generality suppose that all the nonzero terms of $B$ have the same value and that $B_{0} \neq 0$ (divide out the largest power of $g_{n}$ dividing all the terms). This implies that $B_{j}=0$ whenever $e_{n} \nmid j$. With $s$ as above we may pick $b \in F[x]_{\operatorname{deg} g_{n}}$ with $w\left(b B_{0} s^{f_{n}}\right)=0$. Then all nonzero terms of

$$
s^{f_{n}} b B=\sum_{j<f_{n}} B_{j e_{n}} s^{f_{n}-j} b\left(s g_{n}^{e_{n}}\right)^{j}
$$

have the same value, namely, $w\left(B_{0} s^{f_{n}} b\right)=0$. Hence $H(Y):=\sum_{j<f_{n}} \tau\left(B_{j e_{n}} s^{f_{n}-j} b\right) Y^{j}$ is a nonzero polynomial over $\Phi_{n}\left(k_{n}\right)$ of degree less than $f_{n}$, the degree of the minimal polynomial $\Phi_{n}(G)$ of $\tau\left(s g_{n}^{e_{n}}\right)$ over $\Phi_{n}\left(k_{n}\right)$. Hence $0 \neq H\left(\tau\left(s g_{n}^{e_{n}}\right)\right)$, and therefore

$$
0=w\left(\sum_{j<f_{n}} B_{j e_{n}} s^{f_{n}-j} b\left(s g_{n}^{e_{n}}\right)^{j}\right)=w\left(s^{f_{n}} b B\right) .
$$

Thus $w(B)=-w\left(s^{f_{n}} b\right)=w\left(B_{0}\right)$, which was to be proved. This completes the proof of (B) in the case $i=n$ and hence the proof of the Proposition.

Remark 7. Let $w$ be as in Proposition 5. We record here some corollaries of the proof of Proposition 5 above. First, $e_{w_{n+1} / v}=e_{0} \cdots e_{n}$ and $w_{n+1} F[x]=v F+\sum_{0 \leq i \leq n} \mathbb{Z} q_{i}$. Next, if we treat the $\bar{F}$-homomorphisms $\Phi_{i}: k_{i} \rightarrow k$ as identifications, then whenever $0 \leq i \leq n$ we can regard $k_{i}$ as a subfield of $k_{i+1}$ and the extension $k_{i+1} / k_{i}$ is generated over $k_{i}$ by a root of the polynomial (1) of Definition 2.

\section{$\S 3$. Proof of Theorem 1}

Let $\beta$ denote a root of $h$ in some algebraic extension of $K$. Since $(F, v)$ is Henselian, $v$ has unique extensions to $F(\alpha)$ and to $F(\beta)$, both of which we also denote by $v$. We let $w_{\alpha}$ and $w_{\beta}$ denote the extensions of $v$ to $F[x]$ taking each $f \in F[x]$ to $v(f(\alpha))$ and $v(f(\beta))$, 
respectively. The idea of the proof is that the Proposition of Section 2 can be applied to $w_{\alpha}$ and $w_{\beta}$ to obtain an injective homomorphism of short exact sequences

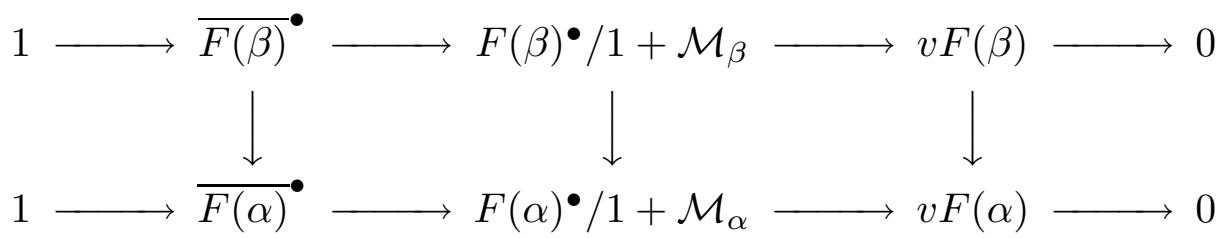

where $\mathcal{M}_{\alpha}$ and $\mathcal{M}_{\beta}$ are the maximal ideals of the valuation rings of $F(\alpha)$ and $F(\beta)$ and where for any field $E$ we let $E^{\bullet}$ denote the multiplicative group of nonzero elements of $E$. This is sufficient to guarantee that $F(\beta)$ is $F$-isomorphic to a subfield of $F(\alpha)$, and hence that $h$ has a root in $F(\alpha)$. A convenient vehicle for expressing the argument formally is the functor $\Delta$ of Brown and Harrison (1970), which we now review.

Let $S(F, \Gamma, r)$ denote the generalized Laurent series field with residue class field $F$, value group $\Gamma$, and symmetric factor set $r$, so $S(F, \Gamma, r)$ consists of formal sums $\sum_{\gamma \in \Gamma} c_{\gamma} t^{\gamma}$ with well-ordered support $\left\{\gamma \in \Gamma: c_{\gamma} \neq 0\right\}$ (Schilling, 1950, pp. 23-24); if the factor set is trivial we write $S(F, \Gamma)$ for $S(F, \Gamma, 1)$. For any valued field $(E, w)$ we let $\Delta E=B / \mathfrak{b}$ where

$$
B=B_{E}=\left\{\sum b_{\gamma} t^{\gamma} \in S(E, w E): w\left(b_{\gamma}\right) \geq \gamma \quad \forall \gamma \in w E\right\}
$$

and

$$
\mathfrak{b}=\mathfrak{b}_{E}=\left\{\sum b_{\gamma} t^{\gamma} \in S(E, w E): w\left(b_{\gamma}\right)>\gamma \quad \forall \gamma \in w E\right\}
$$

and we let $\Delta w: B / \mathfrak{b} \longrightarrow w E \bigcup\{\infty\}$ be defined by the formula

$$
\Delta w\left(\sum b_{\gamma} t^{\gamma}+\mathfrak{b}\right)=\inf \left\{\gamma \in w E: w\left(b_{\gamma}\right)=\gamma\right\}
$$

(setting $\inf \phi=\infty)$. Then $\Delta(E, w):=(\Delta E, \Delta w)$ is a valued field (Brown and Harrison, 1970 ), and $\Delta$ is a functor on the category of valued fields. (Here, morphisms $(E, w) \longrightarrow$ 
$\left(E^{\prime}, w^{\prime}\right)$ are pairs $\left(\varphi, \varphi^{*}\right)$ where $\varphi: E \longrightarrow E^{\prime}$ is a homomorphism and $\varphi^{*}: w E \longrightarrow w^{\prime} E^{\prime}$ is an injective order homomorphism with $\varphi^{*} w=w^{\prime} \varphi ; \Delta$ acts on morphisms in the obvious way.)

Since $h \in \mathcal{P}$ we may assume that $h=g_{n+1}$ where $g$ is a strict system of polynomial extensions of length $n+1$ as in Section 2, whose notation we use here and below. Thus $\gamma_{h}=\gamma_{n+1}$ (cf. Remark 6(B)). Also by Proposition 5(C), $w_{\beta}=w_{n+1}$. Finally, we set $w=w_{\alpha}$, so that $w$ satisfies the hypotheses of Proposition 5.

It is convenient to set

$$
\mathcal{C}=F[x]_{\operatorname{deg} h} \bigcup\left\{a g^{\sigma}: a \in F, 0 \leq \sigma \in \mathbb{Z}^{n+1}\right\} .
$$

By parts (B) and (D) of the Proposition there exists an $\bar{F}$-homomorphism $\varphi: k_{n+1} \longrightarrow k$ such that $\varphi \tau_{n+1}$ and $\tau$ agree on $\{f \in \mathcal{C}: w(f) \geq 0\}$. Also by (A) and (B) of the Proposition for all $f \in \mathcal{C}$ we have $w(f)=w_{n+1}(f)$, and so

$$
w_{n+1} F[x]=v F+\sum_{0 \leq i \leq n} \mathbb{Z} q_{i} \subseteq w F[x] \subseteq \mathbb{Q} v F .
$$

Now let $T$ be a system of representatives in $F$ for $v F$, so that for each $\gamma \in v F$ there exists a unique $a_{\gamma} \in T$ with $v\left(a_{\gamma}\right)=\gamma$. By formula (3)

$$
T_{\beta}:=\left\{a g^{\sigma}: a \in T, 0 \leq \sigma \in \mathbb{Z}^{n+1}, \sigma(i)<e_{i} \forall i \leq n\right\}
$$

is a system of representatives in $F[x]$ for $w_{n+1} F[x]$. Since $w(f)=w_{n+1}(f)$ for all $f \in T_{\beta}$, there is a system of representatives $T_{\alpha} \supseteq T_{\beta}$ in $F[x]$ for $w F[x]$. For each $\gamma \in w F[x]$ let $A_{\gamma}$ denote the unique element of $T_{\alpha}$ with $w\left(A_{\gamma}\right)=\gamma$. Thus $T_{\beta}=\left\{A_{\gamma}: \gamma \in w_{n+1} F[x]\right\}$ and $A_{\gamma}=a_{\gamma}$ for all $\gamma \in v F$. 
These observations on $F[x]$ let us establish connections between $F(\alpha)$ and $F(\beta)$. After all, we may identify $v F(\alpha)$ with $w F[x] ; v F(\beta)$ with $w_{n+1} F[x] ; \overline{F(\alpha)}$ with $k$; and $\overline{F(\beta)}$ with $k_{n+1}$. Thus we will regard $\varphi$ as an $\bar{F}$-homomorphism from $\overline{F(\beta)}$ to $\overline{F(\alpha)}$ with $\varphi(\overline{f(\beta)})=\overline{f(\alpha)}$ for all $f \in \mathcal{C}$ with $w(f) \geq 0$. Similarly we have $v F(\beta) \subseteq v F(\alpha)$ and $v(f(\alpha))=v(f(\beta))$ for all $f \in \mathcal{C}$. Finally, $T_{\alpha}^{*}:=\left\{f(\alpha): f \in T_{\alpha}\right\}$ and $T_{\beta}^{*}:=\left\{f(\beta): f \in T_{\beta}\right\}$ are systems of representatives in $F(\alpha)$ and $F(\beta)$ for $v F(\alpha)$ and $v F(\beta)$, respectively. Indeed for any $\gamma \in v F(\beta)$, the elements of $T_{\beta}, T_{\alpha}^{*}$ and $T_{\beta}^{*}$ of value $\gamma$ (under the valuations $w_{n+1}$, $v$, and $v)$ are $A_{\gamma}, A_{\gamma}(\alpha)$ and $A_{\gamma}(\beta)$, respectively.

The systems of representatives $T_{\alpha}^{*}$ and $T_{\beta}^{*}$ yield symmetric factor sets $r_{\alpha}: v F(\alpha) \times v F(\alpha) \longrightarrow \overline{F(\alpha)}^{\bullet}$ and $r_{\beta}: v F(\beta) \times v F(\beta) \longrightarrow \overline{F(\beta)}^{\bullet}$. For example, for all $\delta, \gamma \in v F(\alpha)$ we have

$$
r_{\alpha}(\delta, \gamma)=\overline{A_{\delta}(\alpha) A_{\gamma}(\alpha) A_{\delta+\gamma}(\alpha)^{-1}}
$$

As in (Brown and Harrison, 1970, Proposition, p. 372) we have an isomorphism

$$
\Theta_{\alpha}: \Delta F(\alpha) \longrightarrow S\left(\overline{F(\alpha)}, v F(\alpha), r_{\alpha}\right)
$$

mapping each formal sum $\sum b_{\gamma} t^{\gamma}+\mathfrak{b}_{F(\alpha)}$ to $\sum \overline{b_{\gamma} A_{\gamma}(\alpha)^{-1}} t^{\gamma}$, and an analogous isomorphism

$$
\Theta_{\beta}: \Delta F(\beta) \longrightarrow S\left(\overline{F(\beta)}, v(\beta), r_{\beta}\right)
$$

Suppose that $\delta, \gamma \in v F(\beta)$ and set $\rho=\delta+\gamma$. Then since any product of the $A_{\mu}$ (for 20 
$\mu \in v F(\beta))$ is in $\mathcal{C}$, we have

$$
\begin{aligned}
\varphi\left(r_{\beta}(\delta, \gamma)\right) & =\varphi\left(\overline{A_{\delta}(\beta) A_{\gamma}(\beta) A_{\rho}(\beta)^{-1}} \overline{A_{-\rho}(\beta) A_{-\rho}(\beta)^{-1}}\right) \\
& =\varphi\left(\overline{\left(A_{\delta} A_{\gamma} A_{-\rho}\right)(\beta)}\right)\left(\varphi\left(\overline{\left(A_{\rho} A_{-\rho}\right)(\beta)}\right)\right)^{-1} \\
& =\overline{\left(A_{\delta} A_{\gamma} A_{-\rho}\right)(\alpha)} \overline{\left(A_{\rho} A_{-\rho}\right)(\alpha)}-1 \\
& =\overline{A_{\delta}(\alpha) A_{\gamma}(\alpha) A_{\rho}(\alpha)^{-1}}=r_{\alpha}(\delta, \gamma) .
\end{aligned}
$$

It follows that $\varphi$ induces a homomorphism of valued fields

$$
\Phi: S\left(\overline{F(\beta)}, v F(\beta), r_{\beta}\right) \longrightarrow S\left(\overline{F(\alpha)}, v F(\alpha), r_{\alpha}\right)
$$

taking each formal sum $\sum b_{\gamma} t^{\gamma}$ to $\sum \varphi\left(b_{\gamma}\right) t^{\gamma}$.

Combining $\Phi$ with the isomorphisms $\Theta_{\alpha}$ and $\Theta_{\beta}$ above yields a $\Delta F$-homomorphism

$$
\Upsilon_{0}: \Delta(F(\beta), v) \longrightarrow \Delta(F(\alpha), v)
$$

We now extract some arguments from (Brown and Harrison, 1970) (which do not depend on the hypothesis there that $(F, v)$ is maximally complete) to show that the existence of $\Upsilon_{0}$ implies the existence of an $F$-homomorphism $F(\beta) \longrightarrow F(\alpha)$. This of course implies that there exists a root of $h$ in $F(\alpha)$, and hence it will complete the proof of the Theorem.

By hypothesis $F(\alpha)$ and $F(\beta)$ are tamely ramified over $F$ (apply Remark 6(A) and Proposition 5(C) with $\mathrm{i}=\mathrm{n})$. Hence both are separable extensions of $F$. (If $F(\alpha)$ is not a separable extension of $F$, then there exists a proper, purely inseparable and tamely ramified extension $F(\alpha) / E$. The residue class field of $F(\alpha)$ is therefore both separable and inseparable over that of $E$, so the residual degree is one. Similarly the ramification index 
is one, and this contradicts that the field extension is proper and tamely ramified. The argument for $F(\beta)$ is the same.) Hence $F(\alpha)$ and $F(\beta)$ are contained in a Galois extension $L$ of $F$. Let $G$ denote the large ramification group of $L / F$, so $G$ is the set of $\sigma \in \operatorname{Gal}(L / F)$ with $v(\sigma(a)-a)>v(a)$ for all $a \in L^{\bullet}$. Our hypothesis that $(F, v)$ is Henselian implies that $G$ is a normal subgroup of $\operatorname{Gal}(L / F)$, so $L^{G} / F$ is a Galois field extension. That $(F, v)$ is Henselian also implies that the extension $L^{G} / F$ is tamely ramified (Zariski and Samuel, 1960, pp. 67-78). Further, $L^{G}$ contains every tamely ramified subextension $E / F$ of $L / F$, including both $F(\alpha)$ and $F(\beta)$. After all, the large ramification group $H$ of an extension $L / E$ with $E / F$ tamely ramified is clearly contained in $G$, but the extension $L^{H}$ of $L^{G}$ cannot be proper because it is both tamely ramified (since $L^{H} / E$ and $E / F$ are both tamely ramified) and wildly ramified (since it is a subextension of $L / L^{G}$, cf. (Zariski and Samuel, 1960, pp. 67-78)). Thus we may as well suppose that $L / F$ is tamely ramified and $G$ is trivial. It follows that the natural homomorphism arising from the functor $\Delta$, call it

$$
D: \operatorname{Gal}(L / F) \longrightarrow \operatorname{Gal}(\Delta L / \Delta F),
$$

is injective (one checks directly that in general the kernel is the large ramification group $G$ of $L / F)$. Thus $D$ is surjective, since both the domain and codomain of $D$ have order $[L: F]=$ $[\bar{L}: \bar{F}](v L: v F)=[\Delta L: \Delta F]$. By Galois theory the $\Delta F$-homomorphism $\Upsilon_{0}$ extends to an automorphism $\Upsilon \in \operatorname{Gal}(\Delta L / \Delta F)$ with $\operatorname{Gal}(\Delta L / \Delta F(\alpha)) \subseteq \Upsilon \operatorname{Gal}(\Delta L / \Delta F(\beta)) \Upsilon^{-1}$. One checks that $D$ maps $\operatorname{Gal}(L / F(\alpha))$ onto $\operatorname{Gal}(\Delta L / \Delta F(\alpha))$ and similarly for $F(\beta)$. Applying the isomorphism $D^{-1}$ we therefore have

$$
\operatorname{Gal}(L / F(\alpha)) \subseteq D^{-1}(\Upsilon) \operatorname{Gal}(L / F(\beta))\left(D^{-1}(\Upsilon)\right)^{-1}
$$


Hence $F(\beta)$ is $F$-isomorphic to a subfield of $F(\alpha)$. Theorem 1 is proved.

Remark 8. We now argue that $\gamma_{h}$ is best possible in Theorem 1 and in fact in a superficial generalization of Theorem 1, in which we replace the hypothesis that $(F, v)$ is Henselian by the hypothesis that $(K, u)$ is finite degree and tamely ramified over a Henselization of $(F, v)$. This fact gives another characterization of $\gamma_{h}$ independent of any strict system of polynomial extensions in which $h$ happens to appear. Without loss of generality $h$ is not linear, and hence we may assume that $h=g_{n+1}$ for some strict system of polynomial extensions $g$ over $(F, v)$ as in Definition 2. Then $g$ can be modified to give a strict system of polynomial extensions over $\left(F_{H}, v_{H}\right)$ where $\left(F_{H}, v_{H}\right)$ is a Henselization of $(F, v)$ by simply replacing each valuation $w_{i}$ by the unique extension $w_{i}^{*}$ of $v_{H}$ to $F_{H}[x]$ with $w_{i}^{*}\left(g_{i}\right)=\infty$. (Uniqueness follows from the fact that an extension $(E, u)$ of $(F, v)$ by a root of $g_{i}$ has degree $f_{u / v} e_{u / v}$ (Proposition 5(C)), and hence the same is true for an extension of $\left(F_{H}, v_{H}\right)$.) Let $\xi$ be a root of $g_{n}$ in a field extension of $F_{H}$. Then $F_{H}[\xi]$ is a tamely ramified finite degree extension of $F_{H}$ (say with valuation $u$ ) with $u(h(\xi))=w_{n}\left(g_{n+1}\right)=\gamma_{n+1}=\gamma_{h}$. However, $F_{H}[\xi]$ cannot contain a root of $h$ since its degree over $F_{H}$ is $\operatorname{deg} g_{n}$, which is strictly less than the degree of the irreducible polynomial $h=g_{n+1}$ over $F_{H}$.

\section{REFERENCES}

Brown, R. (2007), Roots of generalized Schönemann polynomials in Henselian extension fields, preprint. Brown, R. (1972), Valuations, primes and irreducibility in polynomial rings and rational function fields, Trans. Amer. Math. Soc., 174:451-488.

Brown, R., Harrison, D. K. (1970), Tamely ramified extensions of linearly compact fields, J. Algebra, 15:371-375.

Engler, A. J., Prestel, A. (2005), Valued Fields, Berlin: Springer-Verlag.

Khanduja, S., Saha, J. (1997), On a generalization of Eisenstein's irreducibility criterion, Mathematika, 44:37-41.

Schilling, O. F. G. (1950), The Theory of Valuations, Amer. Math. Soc. Math. Surveys No. 4. Providence: Amer. Math. Soc. 
Zariski, O., Samuel, P. (1960), Commutative Algebra, v. II, Princeton: Van Nostrand. 